\title{
Effect of UVB irradiation on microRNA expression in mouse epidermis
}

\author{
BING-RONG ZHOU, YANG XU and DAN LUO
}

Department of Dermatology, The First Affiliated Hospital of Nanjing Medical University, Nanjing 210029, P.R. China

Received October 14,2011; Accepted December 20, 2011

DOI: $10.3892 / \mathrm{ol} .2012 .551$

\begin{abstract}
The aim of this study was to assess the effects of UVB irradiation on miRNA expression in the mouse epidermis. We determined miRNA expression profiles in the epidermis of UVB irradiated mice and untreated mice, and conducted TargetScan and Gene Ontology analyses to predict miRNA targets. Three miRNAs were downregulated and three were upregulated in the epidermis of UVB irradiated mice compared with untreated mice, and were predicted to be associated with photocarcinogenesis, hypomethylation and apoptosis. miRNAs are potentially involved in the pathogenesis of photodamage, and may aid in the treatment and prevention of UVB-induced dermatoses.
\end{abstract}

\section{Introduction}

Ultraviolet (UV) radiation from the sun, particularly its UVB component (290-320 nm), is the major cause of skin cancer. $\mathrm{UV}$ radiation is also known to elicit various other adverse effects, including erythema, sunburn, inflammation, hyperplasia, hyper-pigmentation, immunosuppression, premature skin aging and photocarcinogenesis $(1,2)$. These cell events are mediated through gene activation or suppression (3). Despite intensive investigations into the regulation of gene expression in skin cells, in the majority of cases the precise molecular events remain to be elucidated. Novel methods should be investigated in this area.

MicroRNAs (miRNAs) are short, non-coding RNAs of approximately 22 nucleotides that are thought to regulate gene expression through sequence-specific base pairing with the 3'-untranslated region (3'-UTR) of target mRNAs. To elucidate the molecular mechanisms underlying photodamage, especially skin carcinogenesis by UVB, miRNA expression profiles in UVB irradiated cells have been investigated using miRNA microarrays (4-6). Guo et al investigated the differen-

Correspondence to: Professor Dan Luo, Department of Dermatology, The First Affiliated Hospital of Nanjing Medical University, Nanjing 210029, P.R. China

E-mail: daniluo2005@yahoo.com.cn

Key words: microRNA, microarray, ultraviolet radiation B, photodamage tial expression profiles of miRNAs in $\mathrm{NIH}_{3} \mathrm{~T}_{3}$ cells in response to UVB irradiation (4). Pothof et al found that miRNA expression changes and stress granule formation were most pronounced within the first hours following UVB irradiation, suggesting that miRNA-mediated gene regulation operates earlier than most transcriptional responses (5). The miRNA response may be related to the DNA damage response and cell proliferation (6). However, no studies are currently available on miRNA profiling in response to UVB irradiation in vivo. In this study, we compared the profiles of miRNA expression in 3 pairs of UVB irradiated and untreated mice epidermis, in order to reveal the specific underlying mechanisms associated with photodamage.

\section{Materials and methods}

Animals and $U V$ light source. Animal care and handling complied with protocols approved by the Nanjing Medical University Institutional Animal Care and Use Committee and employed measures to minimize pain and discomfort. Female C57BL/6 mice (12 weeks old) were obtained from the Chinese Academy of Science, Shanghai SLAC Laboratory Animal Co. (Shanghai, China) and were maintained in a pathogen-free barrier facility at Nanjing Medical University. The source of UVB was a BLE-1T158 (Spectronics Corp., Westbury,NY,USA). A Kodacel filter (TA401/407; Kodak, Rochester, NY, USA) was used to block wavelengths of $<290 \mathrm{~nm}$ (UVC). The UVB dose was quantified using a Waldmann UV meter (model no. 585100; Waldmann Co., VS-Schwenningen, Germany) and $180 \mathrm{~mJ} / \mathrm{cm}^{2}$ of UVB was delivered to the dorsal skin of each mouse.

Animal treatments. The C57BL/6 mice were divided into two groups of three animals. The mice in the control group did not receive any treatment. The mice in the second group received UVB $\left(180 \mathrm{~mJ} / \mathrm{cm}^{2}\right)$. Following $24 \mathrm{~h}$ of UVB irradiation, the dorsal irradiated skin was collected. The epidermis was harvested for analysis by heat separation from the dermis.

RNA isolation and miRNA microarray. Total RNA isolation and the miRNA enrichment procedure were performed using a mirVana miRNA Isolation kit (Ambion, Austin, TX, USA) according to the manufacturer's instructions. RNA concentration was quantified using a NanoDrop spectrophotometer (Thermo Fisher, Waltham, MA, USA). The integrity of the RNA was evaluated using an Agilent 2100 Bioanalyzer 
(Agilent Technologies, Santa Clara, CA, USA). RNA labeling and hybridization on the Agilent miRNA microarray chips were performed using a miRNA labeling reagent and hybridization kit (Agilent Technologies) at $37^{\circ} \mathrm{C}$ for $30 \mathrm{~min}$. Total RNA samples (100 ng) were treated with calf intestine alkaline phosphatase (Takara Bio Inc., Dalian, China), denatured using 100\% DMSO (Sigma-Aldrich, St. Louis, MO, USA) at $100^{\circ} \mathrm{C}$ for $8 \mathrm{~min}$ in a thermal cycler, and then transferred to an ice-water bath to prevent reannealing of the RNA. The RNA samples were then labeled with pCp-Cy3 using T4 RNA ligase (Ambion) and incubated at $16^{\circ} \mathrm{C}$ for $2 \mathrm{~h}$. The labeled samples were hybridized to Agilent mouse miRNA microarrays, which contained probes for 627 mouse miRNAs and 39 mouse viral miRNAs as catalogued in the Sanger Centre Database version 10.1 (http://microrna.sanger.ac.uk). Hybridization was performed in SureHyb chambers (Agilent Technologies) for $24 \mathrm{~h}$ at $55^{\circ} \mathrm{C}$. The microarrays were then washed using Agilent prepared buffers. The microarray images were scanned using the Agilent microarray scanner, and gridded and analyzed using Agilent Feature Extraction Software, version 9.5.1 (Agilent Technologies). Normalization was performed using the per-chip median normalization method and the median array (7).

Quantitative real-time PCR analysis for miRNA expression. Expression levels of mmu-miR-233 and mmu-miR-141 were validated using quantitative real-time PCR (qRT-PCR). Primers for qRT-PCR were synthesized by Invitrogen (Shanghai, China). cDNA synthesis was performed using a miScript Reverse Transcription kit (Qiagen, Hilden, Germany) according to the manufacturer's instructions. qRT-PCR was performed using a miScript SYBR-Green PCR kit (Qiagen, Hilden, Germany). The reactions were incubated in a 96-well optical plate at $95^{\circ} \mathrm{C}$ for $15 \mathrm{~min}$, followed by 40 cycles of $15 \mathrm{sec}$ at $94^{\circ} \mathrm{C}, 30 \mathrm{sec}$ at $55^{\circ} \mathrm{C}$ and $30 \mathrm{sec}$ at $70^{\circ} \mathrm{C}$. Expression analysis was performed in triplicate for each sample. mmu-Actin was used as the normalization control. miRNA expression levels were quantified using an ABI Prism 7300 Sequence Detection System (Applied Biosystems, Foster City, CA, USA).

Target prediction and function analysis. TargetScan software was used to predict miRNA targets. To evaluate the TargetScan target predictions for all single miRNAs, we searched for significantly over-represented Gene Ontology (GO) terms among all target genes for all differential miRNAs separately using GOstat software (http://gostat.wehi.edu.au/cgi-bin/goStat. pl) (8). In brief, the program determines all the annotated GO terms and all the GO terms that are associated (i.e., in the path) with the genes analyzed. It then counts the number of appearances of each GO term for the genes inside the group and for the reference genes. First, we pasted the mouse RefSeq ID of the target genes into the text area, we then chose 'mgi' (Mus musculus) from the available GO gene-association databases. For the remaining options we selected the default values. The majority of significant GO terms usually represent the same subset of genes, since the genes may have several GO annotations that are similar. Fisher's exact test was performed to determine whether the observed difference was significant. For each GO category, this resulted in a P-value whereby the observed counts were due to chance. In addition, pathway
A

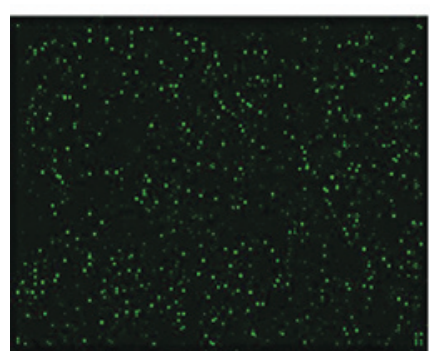

B

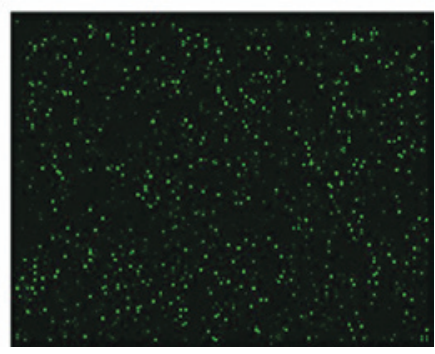

Figure 1. Expression of different miRNAs in mouse skin of two groups detected by microarrays. (A) Untreated mouse epidermis. (B) UVB irradiated epidermis. miRNA, microRNA; UVB, ultraviolet radiation $\mathrm{B}$

analysis of the targets was performed using DAVID Bioinformatics Resources 2008 (http://david.abcc.ncifcrf.gov/).

Statistical analysis. To identify miRNA that was differentially expressed among the groups, a Student's t-test was performed using SPSS version 12.0 (SPSS, Chicago, IL, USA). P $<0.05$ was considered to indicate a statistically significant difference.

The threshold cycle $(\mathrm{Ct})$ value for the genes was determined using SDS software, version 1.2 (Applied Biosystems). The $\mathrm{Ct}$ is the cycle number at which fluorescence is generated as a reaction crosses the threshold. The expression levels of mmu-miR-188-5p, mmu-miR-22, mmu-miR-233, mmu-miR-125a-5p, mmu-miR-146a and mmu-miR-141 were normalized by subtracting their $\mathrm{Ct}$ values from that of the internal control mmu-actin, to obtain $\Delta \mathrm{Ct}$. The $\Delta \Delta \mathrm{Ct}$ method for relative quantitation of gene expression was used to determine the miRNA expression levels. $\Delta \mathrm{Ct}$ was calculated by subtracting the $\mathrm{Ct}$ of actin from the $\mathrm{Ct}$ of the miRNA of interest. $\Delta \Delta \mathrm{Ct}$ was calculated by subtracting the $\Delta \mathrm{Ct}$ of the reference sample (control) from the $\Delta \mathrm{Ct}$ of each sample. Fold change was generated using the equation $2^{\Delta \Delta \mathrm{Ct}}$. Experiments were repeated in triplicate. Statistical significance was measured using the Student's t-test; $\mathrm{p}<0.05$ was considered to indicate a statistically significant difference.

\section{Results}

Significantly differentially expressedmiRNAs. From the miRNA microarray results, six miRNAs (mmu-miR-188-5p, mmu-miR223, mmu-miR-22, mmu-miR-125a-5p, mmu-miR-146a and mmu-miR-141) were found to be differentially expressed in the UVB treatment group compared with the control group $(\mathrm{P}<0.05)$. The microarray images are shown in Fig. 1.

Validation of microarray data by miRNA-specific $q R T-P C R$ analysis. To confirm the microarray findings, we measured the expression levels of six differentially expressed miRNAs (mmu-miR-188-5p, mmu-miR-223, mmu-miR-22, mmu-miR-125a-5p, mmu-miR-146a and mmu-miR-141), using qRT-PCR. The expression of mmu-miR-188-5p, mmu-miR-223 and mmu-miR-22 was increased in the UVB-treated epidermis compared with the control. mmu-miR-125a-5p, mmu-miR-146a and mmu-miR-141 were downregulated following UVB irradiation. These results suggest that the expression levels of the 
Table I. Differentially expressed miRNAs and putative targets.

\begin{tabular}{llll}
\hline & \multicolumn{1}{c}{ miRNA } & Published targets & \multicolumn{1}{c}{ Putative targets } \\
\hline $\begin{array}{l}\text { Three miRNAs expressed } \\
\text { significantly more in UVB group } \\
\text { than in control group }\end{array}$ & $\begin{array}{l}\text { mmu-miR-188-5p } \\
\text { mu-miR-223 } \\
\text { Thu-miR-22 }\end{array}$ & Mef2c & RSPO3, PHF3, RNF144A, ZFP91, RAP2C \\
$\begin{array}{l}\text { Three miRNAs expressed } \\
\text { significantly less in UVB group } \\
\text { than in control group }\end{array}$ & $\begin{array}{l}\text { mmu-miR-125a-5p } \\
\text { mul-miR-146a }\end{array}$ & & FUT9, CLIC4, SNX30, PTGS1, TET2 \\
\hline
\end{tabular}

miRNA, microRNA; UVB, ultraviolet radiation B.

Table II. Gene Ontology (GO) terms for the target genes of treatment responsive miRNAs.

\begin{tabular}{llll}
\hline & GO term & \multicolumn{1}{c}{ Function } & P-value \\
\hline Three miRNAs expressed & 0043283 & Biopolymer metabolic process & $3.64 \mathrm{E}-18$ \\
significantly more in UVB group & 0050794 & Regulation of cellular process & $3.64 \mathrm{E}-18$ \\
than in control group & 0050789 & Regulation of biological process & $3.56 \mathrm{E}-17$ \\
& 0065007 & Biological regulation & $3.56 \mathrm{E}-17$ \\
& 0031323 & Regulation of cellular metabolic process & $4.37 \mathrm{E}-15$ \\
Three miRNAs expressed & 0043283 & Biopolymer metabolic process & $9.13 \mathrm{E}-16$ \\
significantly less in UVB group & 0050789 & Regulation of biological process & $3.06 \mathrm{E}-13$ \\
than in control group & 0005515 & Protein binding & $3.06 \mathrm{E}-13$ \\
& 0050794 & Regulation of cellular process & $3.06 \mathrm{E}-13$ \\
& 0010468 & Regulation of gene expression & $3.06 \mathrm{E}-13$ \\
\hline
\end{tabular}

miRNA, microRNA; UVB, ultraviolet radiation B.

four miRNAs observed in the arrays were consistent with those observed using qRT-PCR (Fig. 2).

Target prediction and function analysis of differentially expressed miRNA. The prediction of miRNA-regulated gene targets is a crucial step in understanding the functions of miRNA. We used TargetScan to obtain predicted gene targets for all differentially expressed miRNAs. As expected, these miRNA genes could potentially regulate several hundred targets. We then examined the significant GO categories and Kyoto Encyclopedia of Genes and Genomes pathways (KEGG) (Tables I-III).

\section{Discussion}

Our present study revealed miRNAs that are sensitive to UVB and baicalin treatment. We analyzed skin tissues from mice in two groups (those irradiated with UVB and controls) 24-h post-irradiation, using an miRNA microarray platform that was able to assess the expression of 627 mouse miRNAs and 39 mouse viral miRNAs. To select differentially expressed miRNAs from the microarray data, we set a cut-off limit at $\mathrm{p}<0.05$. The differentially expressed miRNAs were mmu-miR-188-5p, mmu-miR-223, mmu-miR-22, mmu-miR-125a-5p, mmu-miR-146a and mmu-miR-141.

Among the UVB downregulated miRNAs, miR-141 has been described as a member of the miR-200 family.
Korpal et al (9) found that the miR-200 family miRNAs inhibit epithelial-mesenchymal transition and cancer cell migration by direct targeting of the E-cadherin transcriptional repressors ZEB1 and ZEB2. These findings suggested that the downregulated expression of miR-141 induced by UVB irradiation is involved in the repression of E-cadherin, thereby enhancing migration and invasion during cancer progression. The base excision repair protein MED1 is a predicted target of mmu-miR-146a. MED1 interacts with the mismatch repair protein MLH1 and has a key role in the maintenance of genomic stability, with dual functions in DNA damage response and repair $(10,11)$. MED1 acts as a thymine and uracil DNA N-glycosylase on T:G and U:G mismatches that occur at cytosine-phosphate-guanine $(\mathrm{CpG})$ methylation sites due to the spontaneous deamination of 5-methylcytosine and cytosine, respectively. This event indicates that MED1 is involved in the removal of methylated DNA (12). Abnormal DNA methylation (including hypermethylation and hypomethylation) is a hallmark of the majority of cancers, including colon, lung, prostate and breast cancers, and contributes to carcinogenesis by silencing tumor suppressor genes, upregulating oncogenes and/or reducing genomic stability $(13,14)$. Mittal et al observed global DNA hypomethylation and reduced maintenance methylation in UV-exposed mouse skin (15). In view of these findings, whether or not the downregulated expression of miR146a contributes to UV-induced DNA hypomethylation via MED1 requires further investigation. 
Table III. Pathway analysis of target genes of treatment responsive miRNAs using DAVID Bioinformatics Resources 2008.

\begin{tabular}{llr}
\hline & \multicolumn{1}{c}{ KEGG pathway } & P-value \\
\hline Three miRNAs expressed & ErbB signaling pathway & 9.45 \\
significantly more in UVB group & MAPK signaling pathway & 8.01 \\
than in control group & Dorsoventral axis formation & 6.67 \\
& Prostate cancer & 6.53 \\
Three miRNAs expressed & Chronic myeloid leukemia & 5.94 \\
significantly less in UVB group & Chronic myeloid leukemia & 13.54 \\
than in control group & Notch signaling pathway & 9.32 \\
& Dorsoventral axis formation & 7.83 \\
& MAPK signaling pathway & 6.29 \\
& Pancreatic cancer & 5.67 \\
\hline
\end{tabular}

miRNA, microRNA; UVB, ultraviolet radiation B; KEGG, Kyoto Encyclopedia of Genes and Genomes.

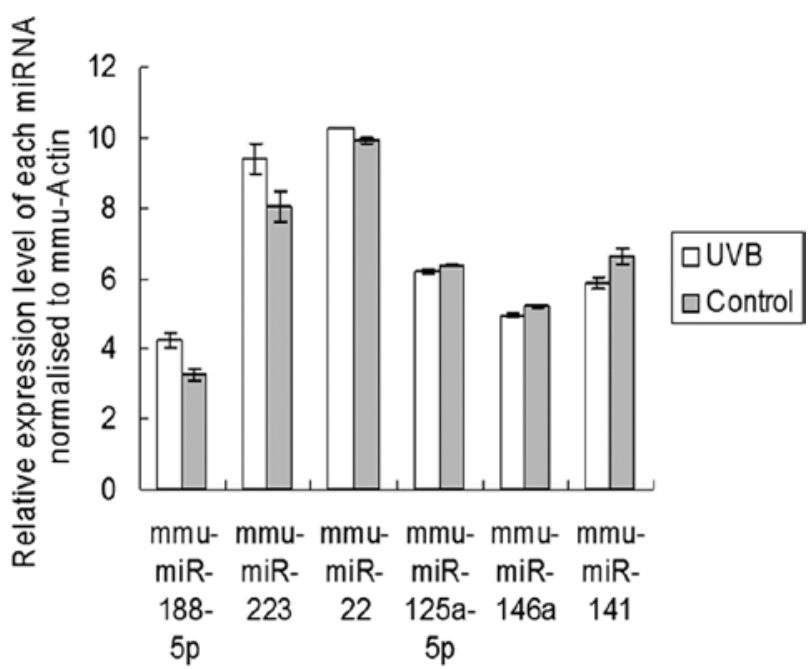

Figure 2. qRT-PCR analysis of dysregulated miRNA expression. UVB, ultraviolet radiation B; qRT-PCR, quantitative real-time PCR; miRNA, microRNA.

In the present study, it is clear that mmu-miR-188-5p, mmu-miR-223 and mmu-miR-22 were upregulated following UVB irradiation. Johnnidis et al found that miR-223 mutant mice have an expanded granulocytic compartment resulting from a cell-autonomous increase in the number of granulocyte progenitors (16). These authors also revealed that Mef2c, a transcription factor that promotes myeloid progenitor proliferation, is a target of miR-223 (16-18). Their data support a model in which miR-223 acts as a fine-tuner of granulocyte production and the inflammatory response. We demonstrated that miR-223 is expressed in the skin of untreated mice, and markedly increases following UVB irradiation. These findings suggest that mmu-miR-223 is sensitive to UVB and elicits a direct or indirect effect of UVB-induced inflammation. RhoB, a predicted target of $\mathrm{miR}-223$, has been reported to protect human keratinocytes from UVB-induced apoptosis through epidermal growth factor receptor signaling (19), suggesting that miR-223 plays a role in regulating keratinocyte survival following UVB exposure. Little is known about the function of miR-188-5p and miR-22.

To further analyze the correlation between patterns of target gene expression and their functional implications, we classified the target genes of all differentially expressed miRNAs into several function categories using GOstat software (http://gostat.wehi.edu.au/cgi-bin/goStat.pl) (8). GO terms concerning the regulation of cellular and biological processes are listed in Table II. The targets of these nine miRNAs were selected for pathway analysis using DAVID Bioinformatics Resources 2008 (http://david.abcc.ncifcrf.gov/) (Table III).

It should be noted that certain miRNAs reported to be involved in the response to UVB irradiation were not observed in this study. For example, miR-21 is known to be involved in the progression of cancer and has been described as an oncogenic miRNA (20), but when it appears together with miR-24, it inhibits growth. Guo et al found that miR-21 and miR-24 appeared together following 12-h exposure to $50 \mathrm{~J} /$ $\mathrm{m}^{2} \mathrm{UVB}$ (4) and their observations of the cell cycle and apoptosis appeared to reveal a sub-G1 DNA content fraction and apoptotic cells 12-h post-irradiation, suggesting that miR-21 and miR-24 together inhibited growth. However, the present study results did not show any changes in miR-21 or miR-24 in the UVB group. This finding may be due to differences in animal selection, UVB dose, chip fabrication or a weakness in the microarray technology.

In conclusion, the focus of this study was to investigate the differential expression profiles of miRNAs in the skin of mice following UVB irradiation. Although our investigation is at a preliminary stage, we believe this study provides a basis for further investigation of the function of expression profiles in miRNAS in signal transduction pathways induced by UVB treatment. miRNAs may therefore be new research hotspots for the prevention or treatment of skin cancer caused by UVB.

\section{Acknowledgements}

This study was supported by grants from the National Natural Science Foundation of China (81000700). 


\section{References}

1. Marrot L and Meunier JR: Skin DNA photodamage and its biological consequences. J Am Acad Dermatol 58: S139-S148, 2008.

2. Timares L, Katiyar SK and Elmets CA: DNA damage, apoptosis and langerhans cells-Activators of UV-induced immune tolerance. Photochem Photobiol 84: 422-436, 2008.

3. Heck DE, Gerecke DR, Vetrano AM and Laskin JD: Solar ultraviolet radiation as a trigger of cell signal transduction. Toxicol Appl Pharmacol 195: 288-297, 2004.

4. Guo L, Huang ZX, Chen XW, Deng QK, Yan W, Zhou MJ, Ou CS and Ding ZH: Differential expression profiles of microRNAs in $\mathrm{NIH}_{3} \mathrm{~T}_{3}$ cells in response to UVB irradiation. Photochem Photobiol 85: 765-773, 2009.

5. Pothof J, Verkaik NS, Hoeijmakers JH and van Gent DC: MicroRNA responses and stress granule formation modulate the DNA damage response. Cell Cycle 8: 3462-3468, 2009.

6. Pothof J, Verkaik NS, van IW, Wiemer EA, Ta VT, van der Horst GT, Jaspers NG, van Gent DC, Hoeijmakers JH and Persengiev SP: MicroRNA-mediated gene silencing modulates the UV-induced DNA-damage response. EMBO J 28 2090-2099, 2009.

7. Liu CG, Calin GA, Meloon B, Gamliel N, Sevignani C, Ferracin M, Dumitru CD, Shimizu M, Zupo S, Dono M, Alder H, Bullrich F, Negrini $M$ and Croce CM: An oligonucleotide microchip for genome-wide microRNA profiling in human and mouse tissues. Proc Natl Acad Sci USA 101: 9740-9744, 2004.

8. Castoldi M, Schmidt S, Benes V, Noerholm M, Kulozik AE, Hentze MW and Muckenthaler MU: A sensitive array for microRNA expression profiling (miChip) based on locked nucleic acids (LNA). RNA 12: 913-920, 2006.

9. Korpal M, Lee ES, Hu G and Kang Y: The miR-200 family inhibits epithelial-mesenchymal transition and cancer cell migration by direct targeting of E-cadherin transcriptional repressors ZEB1 and ZEB2. J Biol Chem 283: 14910-14914, 2008.

10. Bellacosa A: Role of MED1 (MBD4) gene in DNA repair and human cancer. J Cell Physiol 187: 137-144, 2001.
11. Howard JH, Frolov A, Tzeng CW, Stewart A, Midzak A, Majmundar A, Godwin AK, Heslin MJ, Bellacosa A and Arnoletti JP: Epigenetic downregulation of the DNA repair gene MED1/MBD4 in colorectal and ovarian cancer. Cancer Biol Ther 8: 94-100, 2009.

12. Turner DP, Cortellino S, Schupp JE, Caretti E, Loh T, Kinsella TJ and Bellacosa A: The DNA N-glycosylase MED1 exhibits preference for halogenated pyrimidines and is involved in the cytotoxicity of 5-iododeoxyuridine. Cancer Res 66: 7686-7693, 2006.

13. Baylin SB, Esteller M, Rountree MR, Bachman KE, Schuebel K and Herman JG: Aberrant patterns of DNA methylation, chromatin formation and gene expression in cancer. Hum Mol Genet 10: $687-692,2001$.

14. Goodman JI and Watson RE: Altered DNA methylation: a secondary mechanism involved in carcinogenesis. Annu Rev Pharmacol Toxicol 42: 501-525, 2002.

15. Mittal A, Elmets CA and Katiyar SK: Dietary feeding of proanthocyanidins from grape seeds prevents photocarcinogenesis in SKH-1 hairless mice: relationship to decreased fat and lipid peroxidation. Carcinogenesis 24: 1379-1388, 2003.

16. Johnnidis JB, Harris MH, Wheeler RT, Stehling-Sun S, Lam MH, Kirak O, Brummelkamp TR, Fleming MD and Camargo FD: Regulation of progenitor cell proliferation and granulocyte function by microRNA-223. Nature 451: 1125-1129, 2008.

17. Wu X, Li H, Park EJ and Chen JD: SMRTE inhibits MEF2C transcriptional activation by targeting HDAC4 and 5 to nuclear domains. J Biol Chem 276: 24177-24185, 2001.

18. Paroni G, Mizzau M, Henderson C, Del Sal G, Schneider C and Brancolini C: Caspase-dependent regulation of histone deacetylase 4 nuclear-cytoplasmic shuttling promotes apoptosis. Mol Biol Cell 15: 2804-2818, 2004.

19. Canguilhem B, Pradines A, Baudouin C,Boby C,Lajoie-Mazenc I, Charveron $\mathrm{M}$ and Favre G: RhoB protects human keratinocytes from UVB-induced apoptosis through epidermal growth factor receptor signaling. J Biol Chem 280: 43257-43263, 2005.

20. Volinia S, Calin GA, Liu CG, Ambs S, Cimmino A, Petrocca F, Visone R, Iorio M, Roldo C, Ferracin M, et al: A microRNA expression signature of human solid tumors defines cancer gene targets. Proc Natl Acad Sci USA 103: 2257-2261, 2006. 